\title{
New Low-Power 1.5-bit Time-Interleaved MDAC based on MOS Capacitor Amplification
}

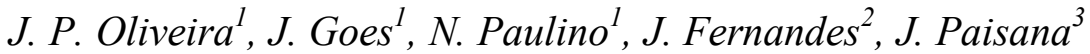 \\ ${ }^{1}$ UNINOVA/ Faculdade de Ciências e Tecnologia \\ Campus da Faculdade de Ciências e Tecnologia \\ 2829-517 Monte da Caparica - PORTUGAL \\ E-mail:.jpao@fct.unl.pt \\ 2 INESC-ID/IST/TU Lisbon \\ Rua Alves Redol, 9 \\ 1000-029 Lisboa - PORTUGAL \\ E-mail: jorge.fernandes@inesc-id.pt \\ ${ }^{3}$ CIEEE/IST/TU Lisbon \\ Av. Rovisco Pais, \\ 1049-001 Lisboa- PORTUGAL \\ E-mail: pcjulio@alfa.ist.utl.pt
}

\begin{abstract}
In this paper a new time-interleaved 1.5-bit MDAC circuit is proposed. This circuit is well suited to be used in ultra low-power high-speed 4-to-8 bits pipeline ADCs. The required gain of two is implemented by switching a MOS capacitor from inversion into depletion within a clock-cycle. Low-power is achieved since no operational amplifiers are required but, instead, simple source-followers are used. Simulation results of a complete front-end stage of a 6-bit 2-channel pipeline ADC demonstrate the efficiency of the proposed technique.
\end{abstract}

\section{INTRODUCTION}

Next generation communications based on impulse radio Ultra-Wideband systems are targeted either for high data rate transfer or ultra-low power low data rate wireless sensor receivers [1]. In these applications, sampling-rates of the order of a few hundred mega-samples per second (MSPS) together with resolutions of about 6 bits are specified for the Analogto-Digital Converters (ADCs) [2].

Pipeline ADCs can achieve very high-speeds with low power dissipation. However, their energy efficiency is much dependent on the optimum resolution-per-stage, on the scaling of the capacitance values and in the residue-amplifier topology used in the multiplying digital-to-analog converters (MDACs) of the pipeline stages. In closed-loop approaches, amplifiers with very high gain-bandwidth products have to be designed which dissipate a significant amount of power.

Parallel pipeline ADCs have been used to achieve medium resolutions at very high sampling rates [3, 4]. Also sharing some common blocks between two or more parallel $\mathrm{ADCs}$, in a time-interleaved fashion can reduce the total power. The closed-loop multiply-by-two residue amplifiers usually integrated in the pipeline ADCs can be replaced by open-loop amplifiers [5, 6], reducing global size and power. However, it becomes mandatory to employ either digital gain-calibration [5] or employ replica circuits for implementing global-gain control techniques [7].

The work presented here, shows for the first time, a multiply-by-two residue amplifier (MBTA) implemented by switching a MOS capacitor from inversion into depletion within a clock-cycle (parametric amplification) using a new MOSCAP parametric amplifier configuration. A complete 2channel interleaved 1.5-bit MDAC circuit based on this principle is described. This circuit is well suited for ultra lowpower high-speed 4-to-8 bits pipeline ADCs. Moreover, low- power is achieved since OPAMP based amplifiers are no longer required, and just simple source-followers are used for isolation between stages.

In section II, the principle of the parametric amplification capability of a MOS capacitor (MOSCAP) is shown. In section III, the new 2-channel 1.5-bit MDAC circuit is fully described. Section IV shows the simulation results and Section $\mathrm{V}$ draws the final conclusions.

\section{LOW-GAIN AMPLIFICATION USING A MOS CAPACITOR}

The needed MBTA in 1.5-bit stages of pipeline ADC can be achieved by using the parametric MOS amplification described in $[8,9]$ where a discrete-time amplifier was evaluated. In this amplifier, the gain is set through the reduction of the total equivalent gate capacitance of a single MOSCAP device, while maintaining the total gate charge between the sampling and the amplification phase. As explained in [8, 9], the capacitance reduction of a MOSCAP can be achieved by moving it from inversion into depletion, as result of changing the control voltage $\left(V_{\text {control }}\right)$ applied to the drain from the lower power supply voltage $\left(V_{S S}\right)$ to the positive power supply $\left(V_{D D}\right)$, as shown in Figs. 1 (a) and (b).

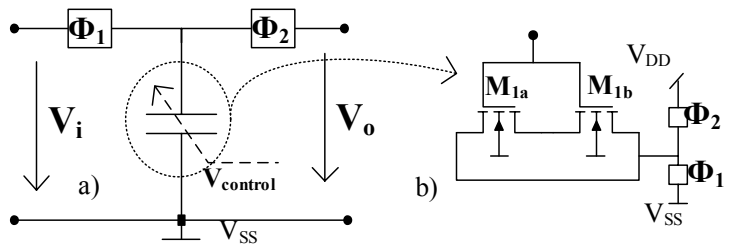

Figure 1. (a) MOSCAP parametric amplifier; (b) MOSCAP implementation using 2 separated devices.

The amplifier circuit operates as follows. During phase $\Phi_{1}$ the input signal is sampled by $\mathrm{M}_{1 \mathrm{a}}-\mathrm{M}_{1 \mathrm{~b}}$ and a corresponding charge is stored. Considering that, in $\Phi_{2}$ both gate charges remain constant (gates are floating), the gate voltage will change by a gain of, approximately, $v_{o} / v_{i}=C_{o x} / C_{g b}$, where $C_{o x}$ is the total gate-oxide capacitance and $C_{g b}$ is the gate-bulk capacitance during the amplification phase [9].

The main differences of the basic MOSCAP structure used here from the one used in $[8,9]$ lies on the fact that 2 halfsized MOSCAPs are used instead of a single one and, on the other hand, their short-circuited sources are left floating. 
The main advantage of this structure is that it decreases the total gate capacitance during the amplification phase since half of the value is left floating. Hence, the total loading capacitance in $\Phi_{2}$ is reduced by half, and amplification gains above 3 can now be easily achieved with an nMOS-type MOSCAP (nMOSCAP) which is of paramount importance to allow the practical implementation of an overall gain of 2 for a complete MDAC. A simulation of the obtainable gain is shown in Fig. 2 by electrical simulations of the circuit shown in Fig. 1.

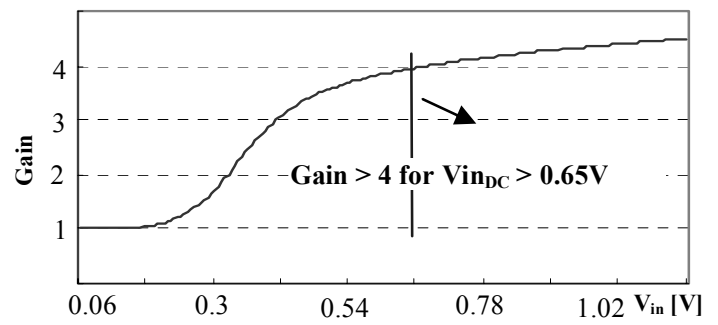

Figure 2. Parametric amplifier gain as a function of the input DC level.

Fig. 2 also shows that the achieved gain depends on the common-mode level of the input voltage reflecting on how well the MOS device is in inversion state during the sampling phase. Therefore an appropriate DC level should be carefully chosen. Another problem to be solved is related with the existing DC level-shifting that occurs when an nMOSCAP $\left(C_{1 N}\right)$ changes from inversion into depletion. To avoid that during the amplification phase and since this level rises above $V_{D D}$, an additional pMOS-type MOSCAP (pMO$\mathrm{SCAP}), C_{2 P}$, should be added as shown in Fig. 3 in order to produce the opposite effect (negative DC level shifting).

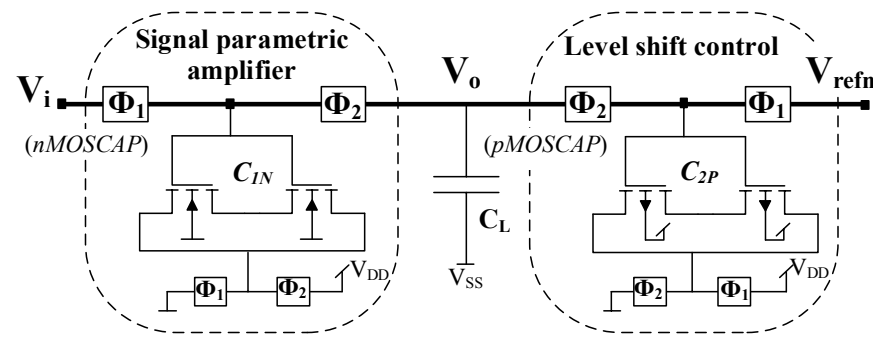

Figure 3. CMOS discrete-time amplifier with output level shift control.

A first-order charge redistribution analysis applied to the described amplifier, results in an equation for the output voltage approximately given by

$$
V_{O} \approx \frac{\alpha_{C 1 n}}{k} V_{i}+\frac{\alpha_{2}-\alpha_{4}}{k} V_{D D}+\frac{\alpha_{4}}{k} V_{r e f n}+\frac{\alpha_{3}}{k} V_{C L, \phi 1}
$$

where $C_{1 N, \phi 1}=\alpha_{C 1 N} \cdot C_{1 N, \phi 2}, C_{2 P, \phi 2}=\alpha_{2} \cdot C_{1 N, \phi 2}, C_{L}=\alpha_{3} \cdot C_{1 N, \phi 2}$ ,$C_{2 P, \phi 1}=\alpha_{4} \cdot C_{1 N, \phi 2}$ and $k=1+\alpha_{2}+\alpha_{3}$.

Parameter $k$ models the gain reduction due to the load capacitance $C_{L}$ and $C_{2 P}$ during phase $\Phi_{2}$. Parameter $\alpha_{C I N}$ reflects the capacitance variation from phase $\Phi_{1}$ to phase $\Phi_{2}$ for $C_{I N}$. Parameter $\alpha_{2}$ represents the relation between $C_{2 P}$, during phase $\Phi_{2}$, and $C_{1 N}$ in phase $\Phi_{1}$. The remaining parameters $\alpha_{3}$ and $\alpha_{4}$ describe the ratio of $C_{L}$ and $C_{2 P}$ in phase $\Phi_{2}$ with respect to the value of $C_{1 N}$ during phase $\Phi_{2}$.
Equation (1) demonstrates that, besides a desired multiplying factor, the output voltage has an offset component partially controlled by the pMOSCAP. It also shows that the load capacitance, $C_{L}$ affects the final gain of the circuit and the output offset level (depending on how the voltage is applied to $C_{L}$ during $\left.\Phi_{1}, V_{C L, \Phi l}\right)$. To reduce this loading capacitance effect, a simple source-follower can be inserted at the parametric output node. This source-follower is also useful to buffer the output when an amplifying stage is loaded by another circuit or amplifier. Using the CMOS distcrete time amplifier represented in Fig. 3 it is possible to design an a 1.5-bit MDAC and then finally a two channel time interleaved 1.5-bit pipeline stage.

\section{1.5-BIT 2-CHANNEL MDAC ARCHITECTURE AND DESIGN}

\section{A. 1.5-bit 2-channel MDAC}

A complete 2-channel time-interleaved 1.5-bit pipeline stage is shown in Fig. 4 where, for simulation purposes, an ideal 1.5-bit quantizer (1.5-bit sub-ADC) is used. The proposed 1.5-bit 2-channel MDAC comprises two fullydifferential MDACs (1 and 2) made of four single-ended MDACs (versions p (Fig. 5) and n named half-MDAC, respectively for the positive and negative signal paths) and two source-follower circuits. As stated before, these buffers are required to isolate the loading effect of the next pipeline stage.

Since the 1.5-bit MDAC operates in opposite phases, the two source-follower circuits are shared across channels. Moreover, this interleaved operation in time becomes possible by the discrete-time operation of the proposed MDACs and meets the requirements for high speed operation. A replica-bias circuit which, will be described later is used to provide the required bias voltage, $V_{\text {bias }}$, for the nMOS current sources in the source-followers.

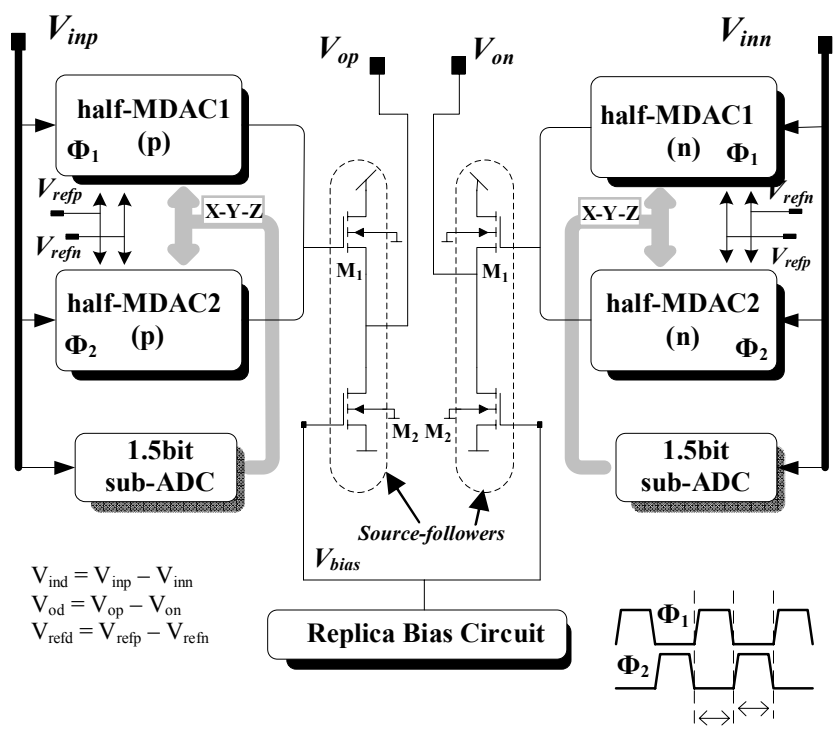

Figure 4. Two-channel time-interleaved 1.5-bit pipeline stage comprising two fully-differential 1.5-bit MDACs. 
The schematic of each 1.5-bit half-MDAC circuit is shown in Fig. 5 (in this case the p-type version is shown). Three switched MOSCAPs, $C_{X}, C_{Y}$ and $C_{Z}$, always operating in inversion have been added. The addition and subtraction function of the 1.5-bit MDAC depends on the values of the 3bit $X, Y, Z$ code (only one bit is enabled in each conversion cycle), which is provided by the 1.5-bit sub-ADC.

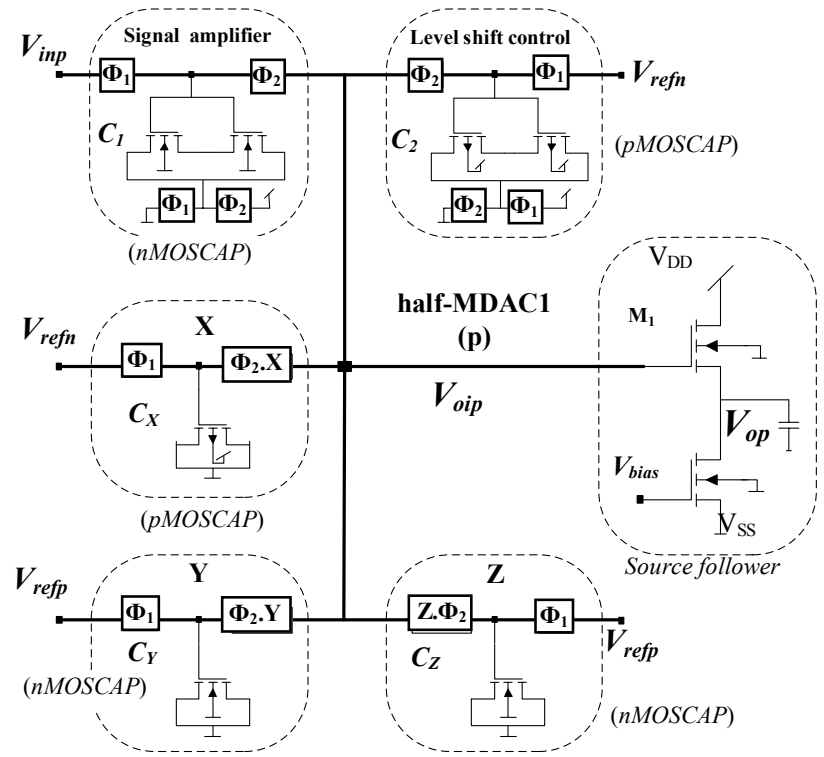

Figure 5. Internal 1.5bit half-MDAC single-ended circuit ( $\mathrm{p}$ version).

During phase $\Phi_{1}$, the input signal is sampled by $C_{1}$, the negative reference voltage $\left(V_{\text {refn }}\right)$ is sampled by $C_{2}$ and by $C_{X}$ and the positive reference voltage $\left(V_{\text {refp }}\right)$ is sampled by $C_{Y}$ and by $C_{Z}$. In the residue-amplification phase $\Phi_{2}$, the capacitance values of $C_{1}$ and $C_{2}$ decrease due to the change from inversion into depletion region, but the capacitance values $C_{X}, C_{Y}$ and $C_{Z}$ remain unchanged. Depending on the value of the $X, Y, Z$ code provided by the 1.5 -bit sub-ADC block, during phase $\Phi_{2}$, only one of the capacitances $C_{X}, C_{Y}$ or $C_{Z}$ is selected to be connected to the input of the corresponding source-follower for charge re-distribution.

Applying, to this half-MDAC, the same methodology used for the parametric amplifier transfer function, results in a similar equation to (1) where new terms are added to take into account the charge re-distribution of $C_{X}, C_{Y}$ and $C_{Z}$. Additionally the $k$ factor also includes the contribution of these MOSCAPs. Considering the differential MDAC implementation, the common-mode components present in (1) are removed, resulting in a final expression for the differential voltage, $V_{o d}$, at the inputs of the source-followers given by:

$V_{o d} \approx \frac{g_{m 1}}{g_{m 1}+g_{s b 1}} \cdot\left(\frac{\alpha_{C 1 n}}{k} V_{i d}-k_{D A C} V_{r e f d} \cdot X+k_{D A C} V_{\text {refd }} \cdot Y+0 \cdot Z\right)$

where $C_{1, \phi 1}=\alpha_{C 1} \cdot C_{1, \phi 2}, \quad C_{2, \phi 2}=\alpha_{2} \cdot C_{1, \phi 2} \quad, \quad C_{L}=\alpha_{3} \cdot C_{1, \phi 2}$ $C_{2, \phi 1}=\alpha_{4} \cdot C_{1, \phi 2} \quad, \quad C_{X Y Z}=\alpha_{5} \cdot C_{1, \phi 2} \quad k=1+\alpha_{2}+\alpha_{3}+\alpha_{5} \quad$ and $k_{D A C}=C_{X Y Z} /\left(C_{1, \phi 2} \cdot k\right)$. Transconductances $g_{m l}$ and $g_{s b l}$ represent, respectively, the main and body-effect transconductances of the main device of the source-follower $\left(\mathrm{M}_{1}\right)$.
In (2), it has been assumed that both half-MDAC have equally sized transistors and $C_{X}, C_{Y}$ and $C_{Z}$ are all nominally equal to $C_{X Y Z}$. A differential MDAC is then composed by two equal size half-MDACs, in which only $X$ and $Y$ signals exchange to implement the n-type version.

Note that, due to the body-effect of the source-followers, their gain is smaller than one. Hence, to compensate this effect the complete 1.5-bit half-MDACs are adjusted and sized to have a gain factor exactly equal to two.

\section{B) Replica-bias circuit output common-mode control}

Another important aspect is that the output commonmode voltage (VCMO) at the outputs of the source-followers can not vary too much in order to avoid DC accumulation errors in the subsequent pipeline stages. A replica-bias circuit as the one depicted in Fig. 6 is used to guarantee that VCMO is restored at the output of every 1.5-bit MDAC and adjusted to a proper value against process and supply variations. Note that a single replica-bias block can be used to bias all MDACs in the pipeline chain. It comprises two timeinterleaved half-MDACs connected to a same replica sourcefollower. The average voltage at the buffer output is set and controlled by a negative feedback loop which includes a very simple amplifier. The objective is to replicate the operation of the differential MDAC and controlling the common mode voltage present at the MDACs buffers outputs through a biasing voltage $V_{\text {bias }}$. As referred before, stabilizing the output common-mode component at the outputs of each stage prevents biasing error propagation along the pipeline. Note that the OTA used here does not need a high bandwidth since it is not inserted in the main signal path. Therefore it can be designed with minimum current.

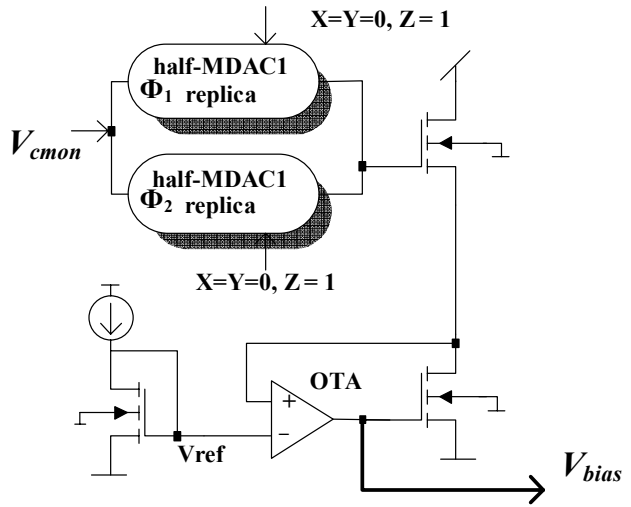

Figure 6. Replica bias block used to generate Vbias for all source-followers.

\section{Simulation RESUltS}

A fully-differential time-interleaved 1.5-bit MDAC has been designed in 130nm 1P-8M CMOS technology for a $1.2 \mathrm{~V}$ supply voltage. This MDAC was specially designed to meet the specifications of a front-end stage for a 2-channel 6bit $250 \mathrm{MS} / \mathrm{s}$ pipeline ADC. The adopted differential fullscale input is $400 \mathrm{mVp}$-p and, to reduce the size of the switches, $1.0 \mathrm{~V}$ and $0.0 \mathrm{~V}$ are used for $V_{\text {refp }}$ and $V_{\text {refn }}$, respectively. The input and output common-mode-voltages are set to $0.65 \mathrm{~V}$ and $0.45 \mathrm{~V}$, respectively. 
For the switches connected to the input signals, asymmetrical CMOS transmission gates with dummy structures are used to minimize signal-dependent charge injection. All remaining switches are made only of single nMOS or pMOS devices. Very small source-followers are used with an input parasitic capacitance smaller than $50 \mathrm{fF}$ and with a driving capability of a fixed load of $500 \mathrm{fF}$.

Capacitors $C_{I N}, C_{2 P}$ and $C_{X Y Z}$ are sized to have, in inversion, a capacitance value of about $600 \mathrm{fF}, 200 \mathrm{fF}$ and $200 \mathrm{fF}$, respectively. These values are then fine-tuned by exhaustive simulations in order to obtain a precise gain of 2.0 in typical conditions and when $Z$ is active. A channel length of $360 \mathrm{~nm}$ is used in all MOSCAPs to achieve low transition times when switching the MOSCAPs from inversion into depletion region (and vice-versa).

Fig. 7 (a), (b) and (c) show the simulated conversion characteristic of the 1.5-bit MDAC for a slow differential input ramp signal and for 3 different process corners. A slow input ramp signal was used to obtain accurate simulations results but the 1.5-bit MDAC is working at full speed (250 $\mathrm{MS} / \mathrm{s})$. Simulated gain error variation at the output is smaller than $3.2 \%(0.4 \%$ of error in typical conditions and when $Z$ is active) for all 3 corners. The complete 2-channel 1.5-bit MDAC core dissipates only $2.37 \mathrm{~mW}$ when operating at 250 $\mathrm{MS} / \mathrm{s}$ and at $1.2 \mathrm{~V}$. The replica bias block dissipates $0.73 \mathrm{~mW}$ and the two source-followers dissipate only $0.49 \mathrm{~mW}$.

\section{CONCLUSIONS}

In this paper a new time-interleaved 1.5-bit MDAC circuit was proposed. This circuit is well suited for ultra low-power high-speed 4-to-8 bits pipeline ADCs. The gain of two was implemented by switching a MOS capacitor from inversion into depletion within a clock-cycle using a new MOSCAP configuration. Low-power was achieved since no operational amplifiers are required but, instead, simple source-followers are used. Simulation results of a complete front-end stage of a 6-bit 2-channel pipeline ADC demonstrated the efficiency of the proposed technique.

\section{ACKNOWLEDGEMENTS}

The research work was partially supported by the Portuguese Foundation for Science and Technology (FCT/MCT) under SIPHASE (POSC/EEA-ESSE/61863/2004), LEADER (PTDC/EEA-ELC/69791/2006) and SPEED (PTDC/EEAELC/66857/2006 ) projects.

\section{REFERENCES}

[1] D. Cabric, et. al.,"Spectrum Sharing Radios", IEEE Circuit and Systems Magazine, pp. 30-45, Vol. 6, no. 2, 2006

[2] P.P. Newaskar, et. al., "A/D precision requirements for an ultrawideband radio receiver", IEEE SIPS'02, pp. 270-275, Oct. 2002.

[3] C. S. G. Conroy, D. W. Cline, and P. R. Gray, "An 8-b 85-MS/s parallel pipeline A/D converter in 1- $\mu \mathrm{m}$ CMOS," IEEE J. Solid-State Circuits, vol. 28, pp. 447-454, Apr. 1993.

[4] S. Gupta, et. al., "A 1GS/s 11b Time-Interleaved ADC in 0.13um CMOS” Proc. IEEE ISSCC Dig. Tech. Papers, pp. 576-577, Feb. 2006.

[5] B. Murmann and B. E. Boser, "A 12-bit $75 \mathrm{Ms} / \mathrm{s}$ pipelined ADC using open-loop residue amplifier," IEEE J. Solid-State Circuits, vol. 38, no. 12, pp. 2040-2050, Dec. 2003.
[6] Ding-L. Shen, Tai-C. Lee, “A 6-bit 800-MS/s Pipelined A/D Converter With Open Loop Amplifiers", IEEE J. Solid-State Circuits, vol. 42, no. 2, pp. 258-268, Feb. 2007.

[7] K. Poulton, R. Neff, A. Muto, W. Liu, A. Burstein, and M. Heshami, "A 4 Gsample/s 8b ADC in $0.35 \mu \mathrm{m}$ CMOS," in IEEE ISSCC Dig. Tech. Papers, pp. 166-457, Feb. 2002.

[8] S. Ranganathan, Y. Tsividis, "A MOS capacitor-based discrete-time parametric amplifier with $1.2 \mathrm{~V}$ output swing and $3 \mathrm{uW}$ power dissipation", IEEE ISSCC. Dig. Tech. Papers, pp. 406-407, Feb. 2003.

[9] S. Ranganathan, Y. Tsividis, "Discrete-Time parametric amplification based on a three-terminal MOS varactor: analisys and experimental results", IEEE, J. Solid-State Circuits, no. 12, pp. 2087-2093, Dec. 2003.
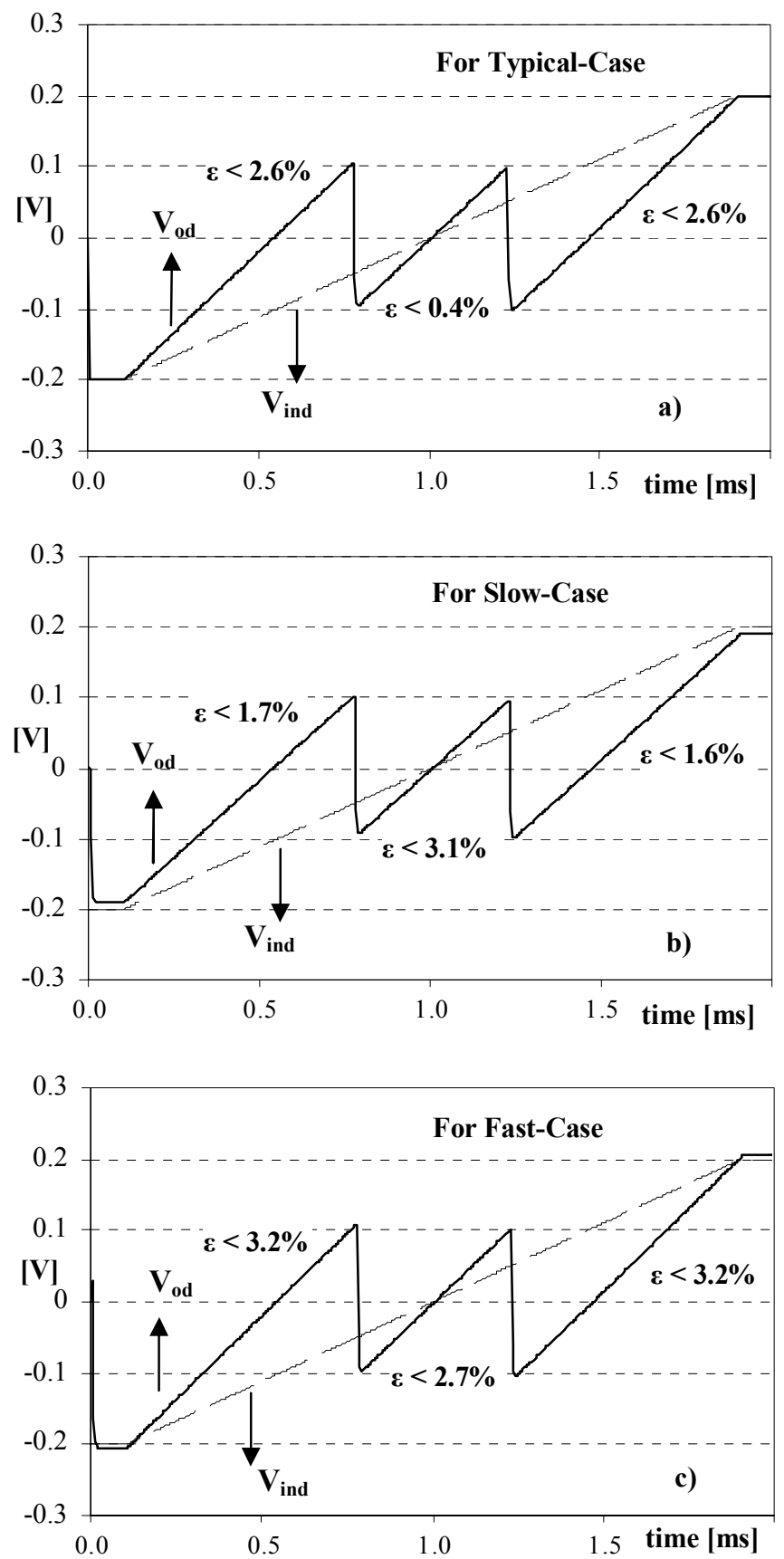

Figure 7: Simulations of the differential output of a 2-channel 1.5-bit MDAC for a differential input ramp signal: a) typical; b) Slow-case; c) Fast-case; 